\title{
MODULAR SYSTEM OF SENSORS FOR MONITORING OF MUSEUMS' INTERNAL ENVIRONMENT
}

\author{
J. Valach*a ${ }^{*}$ K. Juliš $\check{a}^{\mathrm{a}}$ P. Štefcováb \\ a Institute of Theoretical and Applied Mechanics AS CR, v.v.i., Prosecká 76, 19000 Prague 9, Czech Republic, valach@itam.cas.cz \\ b National Museum, Václavské námůstí 68, 11579 Prague 1, Czech Republic, petra_stefcova@nm.cz
}

KEY WORDS: Indoor climate, sensors, monitoring, protection of museum collections

\begin{abstract}
:
The paper outlines basic concepts of the system for indoor climate monitoring being currently developed in a four-year project. The project concentrates on building system of sensors, the related infrastructure for communication between these sensors and the server for centralized data storage and processing. The components of the planned system are highly modular for adjustment to specific requirements of given collection. The project's aim is to provide basis for remote monitoring of indoor climate and environment of exhibitions and depositories of museums, galleries, archives, etc. situated in historical buildings like castles, chateaus, town halls, where the protection of the building limits measures for indoor climate optimization. Finally the expected outcomes envisage the development of specialized sensors mainly for pest control and development of portable version of data sensing units for supervision of environmental parameters of collection during transport.
\end{abstract}

\section{INTRODUCTION}

The supervision and control of indoor climate in museums and other institutions keeping large collections is a key factor in long term preservation of their unique items. Preservation is even more problematic in those historical buildings where the options of applicable protection techniques are further limited by multiple factors concerning conservation issues (Camuffo 2001, Cassar 1995, Huijbregts 2012). The first important step in controlling the environment is the measurement of its parameters. Simultaneously, as a second step, understanding the relation between measured environmental variables and signs of degradation advancement in typical material classes e.g. metal, leather, paper, etc. can refine setting acceptable ranges of decisive climate variables. Both steps constitute noninvasive measures and the combined introduction and assessment of their effectiveness is a major scope of the presented project.

\section{PROJECT DESCRIPTION}

\subsection{Project Goal and Motivation}

The aim of the proposed system is to assemble a comprehensive network including sensing devices placed in the monitored indoor environment, dedicated server storing acquired data and a set of software tools and applications for data processing and evaluation.

This kind of sensor infrastructure is actually being developed in many institutions (e.g. Child 1993, Smoohs 2012). The major differences that distinguish the presented system from other implementations are on one hand the objective to interface existing heterogeneous systems and therefore to minimize necessary investments and on the other hand the attention paid to data processing and mining.

\subsection{Main concept}

The goal of the project is to build modular hardware platform and a unified data exchange structure enabling central processing of remote data with simultaneous supervision of indoor climate parameters and tools for evaluation of recorded data. As mentioned in previous paragraphs, the recorded data can be used not only to control indoor environment, but also for investigation of relationships between decay rate and surrounding environment for specific categories of materials. The project has started in the year 2012 and is expected to be completed by the end of year 2015 .

\subsection{Reuse of an Existing Infrastructure}

At the present time, sensors displaying and registering actual temperature and/or humidity, storing maximum, minimum and mean values are installed in many museums. As mentioned in the introductory part, preinstalled infrastructure can be also utilized for acquisition data without interference to its original function which is to control of indoor environment. These sensors, mainly temperature and humidity ones are used for HVAC (heating ventilation and air conditioning) systems, and also other sensors were installed and operated before start of the project. Some sensors are directly connected to the climate conditioning units, while other store the measured data till operator downloads data manually into another digital medium for further processing. The inconvenience of manually inspect every sensor and transferring data either to computer or to paper records makes the task to be very tedious work. Considering the long term, this state is also economically challenging as museum workers being highly skilled professionals, could curate and help collections more if freed from the duties to record and transmit data which can be easily performed by computers and networks. However digitized data raises some issues too: in many cases stored histories of indoor climate evolution already exists, but data is recorded in incompatible proprietary format and accessible only via commercial software 
limiting possibility to investigate and cross-correlate data from sensors made by various manufacturers.

Therefore the aim is to overcome these drawback and to build reliable and easy to operate system for telemetric data acquisition upon heterogeneous ,ecosystem“ of various new and already installed tools, devices and sensors provided by various manufacturers in such a way that these heterogeneous sources will yield unified data structure.

\section{MAIN COMPONENTS OF THE SYSTEM}

\subsection{General Scheme of the Proposed Network}

The system is by design scalable and its architecture resembles star-like topology. The structure of the system is quite simple; it consists of an array of independent elements for data acquisition communicating with the data server over Internet using standard protocols and custom written software. Data acquisition elements consist of control unit, sensor interface and communication unit. The variability of attachable sensors, as well as communication means makes this element of the system a powerful and universal tool for remote data sensing.

The main reason why the whole device contains separate sensor interface is the requirement to select variables important for given collection individually. Similar argument is also valid for communication unit that is equipped with technologies according to connectivity at a given location. In the block diagram in the Figure 1 the basic components of the sensing part of the system are depicted.

\subsection{Components of Basic Element}

The developed system's basic element is modular, designed to interface variety of sensors. Deployed units can be either powered from mains or by battery. The control unit is able to store data in the case connection to server has been interrupted. Sensors consuming the minute amount of energy and working discontinuously can be considered as the best candidate for this approach. The challenge consists in building them independent on external power supply by harvesting local sources of energy. Sources of energy available on-site, like temperature gradient, wind draft, vibrations (e.g. Glynne-Jones 2004), electromagnetic radiation or illumination or other forms of energy will be considered.

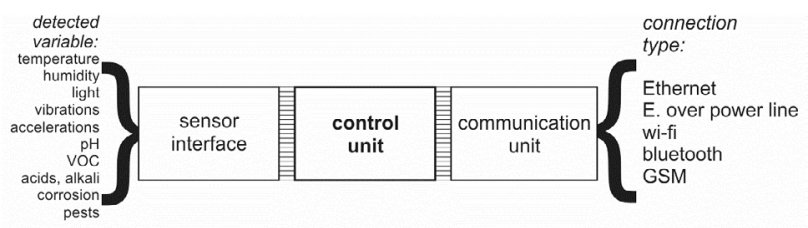

Figure 1: Components of system elements

\subsection{PC Based Unit}

Most important part of the basic element is the control unit. In order to provide room for future scalability and possible functionality upgrade, the central units are not based on dedicated or custom built chips and circuits, but they utilize flexibility of PC based board with chipsets providing the interface to standard and industrial peripherals and sensors. In order to make interfacing to "dumb" sensors possible, it is necessary to provide the control unit with enough computational power, because it would be necessary to convert data mentioned for human information but not for data transfer. These units represent fully equipped PC-compatible devices.

The major advantages of the adopted solution are simplicity of programming and interfacing other devices. In addition to these, it is possible to outline the following benefits:

- Small form factor for easy miniaturization;

- Energy efficient components limiting power consumption and also release of heat, implying that the device can work on backup battery for a long time (weeks) without interrupting its task to acquire and store data;

- $\quad$ Linux based operating system provides a luxury and development throughput of high level programming language in comparison to coding specialized chips in machine code;

- Devices can be accessed and their software can be upgraded or repaired remotely;

- The price of the device is quite low, especially when compared to commercially produced systems;

- Extensive amount of peripherals and add-on boards is available on the market;

- Huge user community providing for help and development assistance.

At the present time, there are known several manufacturers producing these integrated PC board like e.g. Arduino or ALIX (Arduino 2012, ALIX 2012). In this project, ALIX board is used. It is a miniature form factor system board optimized for slim clients and embedded applications.

This unit provides connection to the sensor interface and also to the communication unit. The first prototype is shown on Figure 2.

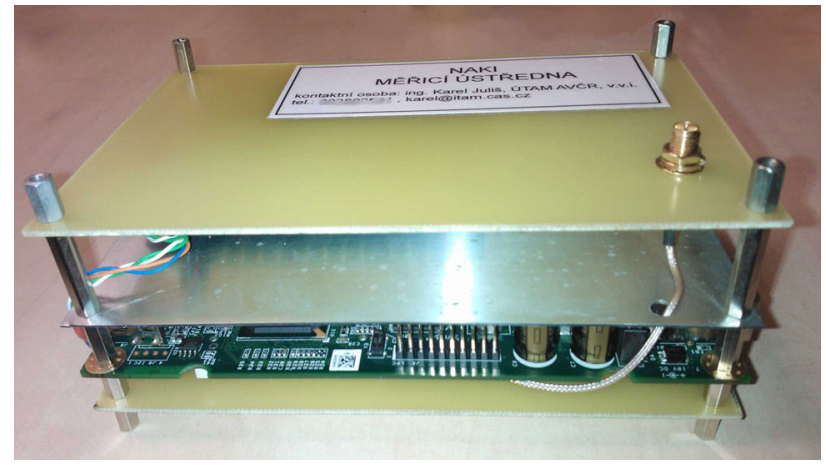

Figure 2: Prototype of the control unit

\subsection{Sensor Interface and Measured Parameters}

The interface to the sensors is compatible with the vast majority of sensors available on the market. This domain is in a fully developed stage and the market offers sensors for any measurable property. Therefore knowing material, composition of collections, decisive parameters and suitable sensors can be easily selected. Database compiling knowledge on dangerous environmental parameters for major types of materials can serve as a starting point in this attempt (Dvoúáková J. 2001).

It was shown that indoor environment can be characterized by several parameters measurable by exploiting various physical and chemical principles. The most common parameters are temperature and relative humidity. Depending on collection type, other important variables are the exposition to light, to ultraviolet radiation or to thermal radiation. It is necessary to distinguish between these three radiations as they trigger different decay processes in objects, based on their composition 
and structure. Another key class of sensors detects corrosive agents in the indoor atmosphere. These devices are based mainly on a vibration member equipped on its surface with a layer of investigated metal. As oxidized or corroded layer builds up it causes the change of its resonant frequency which is measured in some close-loop setting. The presence of dust and other particles in the indoor air is also important parameter. These particles are usually counted for and the distribution of their sizes can be also measured. VOC, volatile organic compounds are considered dangerous especially for organic components which are sensitive to them. Among other detectable properties accelerations, vibrations, chemical properties like various gases, acids, $\mathrm{pH}$ can be named. Where necessary, these sensors will be attached to the sensor interface.

\subsection{Specialized Sensors for Pest Control}

Pest control is an important issue in collections' protection. In order to keep insect infestation low or to eliminate it completely, the collections undergo preventive measures involving regular application of gas toxic to the insect. These measures are adopted even if there is no sign of pest presence. The reason why this crude approach is chosen can be found in the fact, that there are no effective sensors detecting pests' presence in the collections. In order to overcome this situation, specialized sensors based on combination of chemical attraction and image analysis methods are to be developed. Their use would allow taking qualified decision if the gas has to be applied. This would reduce exposure of workers in museums to these potentially harmful substances and also save resources.

\subsection{Communication Unit}

Depending on the situation, the control unit either stores data locally or transmits it to the dedicated server via communication channel available at the given location. The data transfer is provided by communication unit attached to the central unit. The internet connection can be carried out over metallic line, the power lines of AC $220 \mathrm{~V}$ can be also utilized, as well as wi-fi, Bluetooth or GSM modem wireless connection (see e.g. Gungor 2009).

\subsection{Setup for the Use in During Transport}

Transportation is a risky task and represents a serious threat to objects in collections, therefore precautions are necessary. Portable elements of the system can be used to on-line monitor collections during transport and to record whether conditions remain in predetermined range - this applies especially to location, temperature, inclination (allowed orientations range), acceleration and vibration control.

Introduction of sensors recording conditions during transportation can be used as a tool imposing negotiated parameters of collection's transport with companies that provide the service.

\section{SERVER AND SOFTWARE}

\subsection{Hardware Part and Its Main Functions}

Building of the system includes development of dedicated server for data storage, processing and presenting. Data is stored centrally and available on-line for supervision of collections by responsible personnel and also for extensive data-mining. Other requirements laid upon the server are system management, mirroring, backup, uninterrupted operation, hot-swap, support for UPS, etc. The server runs the database server, the web server allowing simultaneous execution of applications, the warning system and the data mining. The main functions of the server are shown on Figure 3.

Central unit stores data records in a uniform structure and processes them "on the fly" for immediate response if data indicate abrupt change or are placed outside acceptable limits. In the long-term the unified and centralized way of storing data will allow to assess what parameters and what thresholds are permissible for collection preservation. Upon combination of models and recorded data, it would be possible to deduce trends of measured parameters and therefore to refine the climate control and to prevent loss of value.

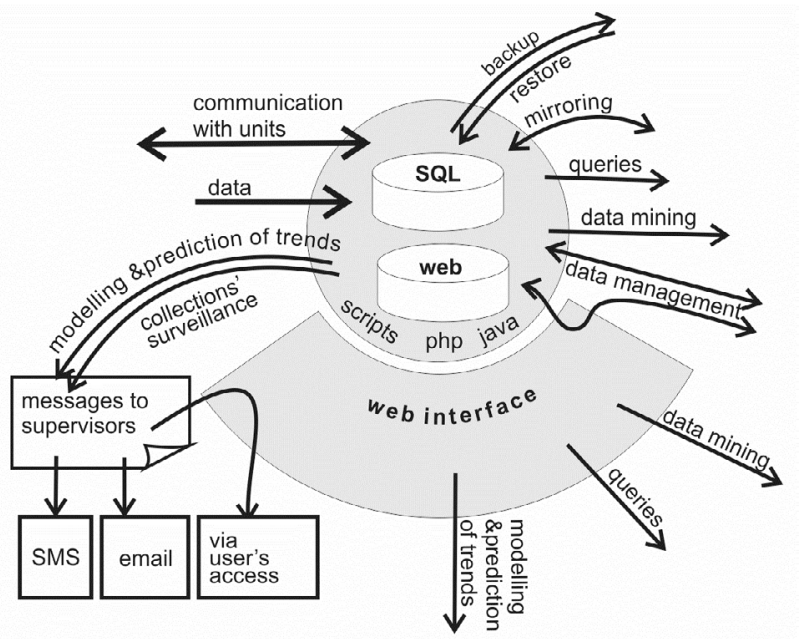

Figure 3: Schematics of major function of system's server

\subsection{Software and Data Processing Part}

Along with hardware development of basic elements and server, data processing applications are to be built. A unified structure of data enables finding characteristic changes in patterns of indoor parameters, which can be related to deterioration of collections' objects.

In contrary to the most cases, where acquired data is used for optimal indoor climate control without being stored for consequent processing, this project can be distinguished from other projects of this kind based on the attention it pays to use and processing of centrally stored data. Unified data structure relying on sequential stream of data records mimicking the way data is acquired by sensors, as well as XML (Extensible Markup Language) metadata for semantic annotation important for computer "understanding of stored data" simplify data processing and evaluation. The record's structure allows for effective selection of studied location, timespan and variables in the first step and investigation of mutual cross-correlation between phenomena in the second step. Dedicated routines watching whether measured values of parameters fit into preset interval deduced from requirements of the most sensitive material in supervised collection are also provided. In the case the threat to the protected collection is detected, this routine triggers sending warning messages to responsible persons via email, sms or user account. Signal from every sensor would be also statistically evaluated to provide user with values representing properties of measured signal like mean, standard deviation, etc.

Design of data structure involves SQL relational databases, unified data format, XML metainformation, tables summarizing information on buildings, rooms, sensors, units and also on collections, their prevailing materials and tables of material 
sensitivity to various environmental variables. Database is to be built following rigorous rules, like exclusion of information redundancy in tables, etc.

A sensible balance in database tables design has to be established between simplicity for easy maintenance and ability to express significant relations in sufficient detail. For example, it is useful to keep information on rooms and control units separately, as there can be unit connecting sensors in two adjacent rooms, as well as two separate units can be placed in the same room. Figure 4 illustrates the situation.

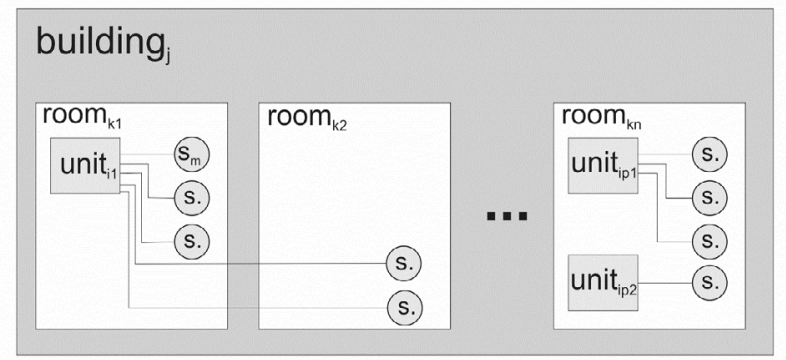

Figure 4: Objects and their relations in the database (museum building, rooms, units and sensors)

\subsection{Data Mining}

The aim of extended data processing and analysis is to investigate correlations between collection's state and recorded parameters of indoor environment. These ad hoc interpretations of records (that play role of observation in laboratory tests) are the only "experiments" allowed to be carried out on objects in collections. Limited museums' budget, especially in such an age of austerity measures, implies that many collections are kept under sub-optimal conditions. Also protection of historical building of museum limits the extent in which indoor climate can be controlled, therefore it can be in conflict with requirements of collection (La Gennusa 2008). This situation is not an immediate threat to the objects, but minor signs of deterioration can appear. The seemingly inactive observation, in fact, successfully assesses the dynamics of deterioration of collections consequently allowing to intervene prior irreparable damage occurs to objects.

\section{DISCUSSION AND CONCLUSIONS}

The final goal of evaluation of stored data would be better understanding of decisive indoor climate parameters from the point of view of collection protection and mitigating decay of its objects.

One of the contemplated applications exploiting the accumulated data would be the adjustment of ranges of safe or acceptable margins of environmental parameters for storage of various types of materials. It would be also possible, in the case of the simplest materials, to combine recorded indoor climate data with so called dose-response functions for rate of degradation prediction (Tidbald 2001). These dose-response functions are determined empirically and relate material degradation rate, like corrosion rate in the case of metals, with concentration of some pollutant or with other parameter, like relative humidity. Similar approach has been adopted for determination of decay of elements of historical buildings as a function of atmospheric pollutants and e.g. rainfall in EUfunded project Cultstrat (Watt 2009).

The importance of comparative studies for understanding deterioration mechanisms of objects in collections is evident.
The investigation of correlation between degradation of materials and objects and the indoor climate allows evaluating sensitivity of studied material on various environmental parameters. It enables museum workers to estimate influence of indoor climate parameters on objects' decay without actually performing any tests destructively. Therefore, in such a way, safe margins of indoor climate preparation can be determined.

\section{REFERENCES}

ALIX PC boards. http://www.pcengines.de (accessed 11 June 2012)

Arduino PC boards, http://www.arduino.cc/ (accessed 11 June 2012)

Camuffo, D. (et al.), 2001. Environmental monitoring in four European museums, Atmospheric Environment 35(Supplement 1), pp127-140.

Cassar, M., 1995. Environmental Management: Guidelines for Museums and Galleries, Routledge: London.

Child, RE (ed.) 1993, Electronic Environmental Monitoring in Museums. Denbigh, Clwyd, Wales: Archetype Publications Ltd.

Dvoůáková J. 2001. Risk factors influencing various types of materials in museum collections, ůtefcová P. (ed.), Preventivní ochrana sbírkovůch půedmůtů (in Czech), 2nd edition, Národní muzeum, Praha, ISSN: 80-7036-129-8

Glynne-Jones, P. (et al.), 2004. An electromagnetic, vibrationpowered generator for intelligent sensor systems, Sensors and Actuators A, 110, pp. 344-349.

Gungor, V.C., 2009. Industrial Wireless Sensor Networks: Challenges, Design Principles, and Technical Approaches, IEEE Transactions On Industrial Electronics, 56 (10).

Huijbregts, Z. (et al.), 2012. A proposed method to assess the damage risk of future climate change to museum objects in historic buildings, Building and Environment, 55.

La Gennusa, M. (et al.), 2008. Conflicting needs of the thermal indoor environment of museums: In search of a practical compromise, Journal of Cultural Heritage, 9.

Smoohs project description, http://www.smoohs.eu/tikiindex.php (accessed 11 June 2012)

Tidbald J. (et al.) 2001. UN ECE ICP Materials: Dose-response functions on dry and wet acid deposition effects after 8 years of exposure. Water, Air and Soil Pollution, 130, pp. 1457-1462.

Watt, J. (Ed.) (et al.) 2009. The Effects of Air Pollution on Cultural Heritage, Springer, ISBN 978-0-387-84892-1.

\section{ACKNOWLEDGEMENTS}

The project "Unified modular system of remote on-line monitoring of environmental parameters of depositories and expositions" is supported by the programme of applied research and development of national and cultural identity (NAKI) of the Ministry of Culture of the Czech Republic - grant No. DF12P01OVV27. The support is gratefully acknowledged. 